\title{
Fiber Content Determination of Linen/Viscose Blends Using NIR Spectroscopy
}

\author{
Jing Huang, ${ }^{\mathrm{a}, \mathrm{c}}$ and Chongwen $\mathrm{Yu}^{\mathrm{a}, \mathrm{b}, *}$
}

\begin{abstract}
Linen/viscose blended yarns provide unique properties, but the quality and cost of the fabric composed of the blended yarns are affected by the amount of linen fibers. The existing methods of detecting blending ratio are microscopy or specific component dissolution, which is time-consuming and inconvenient. This study considers the possibility of rapid and simple determination of linen content by the near infrared (NIR) method. A set of linen/viscose powdered blends with 11 different ratios was fabricated. For each sample, 10 sets of spectra were collected by Fourier transform (FT)-NIR spectrometer. A total of 110 spectra sets were generated, in which 60 were used for calibration and 50 for validation. There were verified differences in NIR peaks assigning to representative chemical bonds in cellulose. With the chemometric analysis, a partial least squares (PLS) model was established to predict the linen content in a blended sample. With a combination of smoothing, baseline offset, and multiplicative scatter correction processing of the spectral data, the established PLS model was further improved to achieve a standard validation error of only $1.182 \%$ and SD value of the predicted linen content less than 0.2 , which indicated the accuracy of the developed method.
\end{abstract}

Keywords: NIR spectroscopy; Linen/viscose blends; Fiber content; PLS model

Contact information: a: College of Textiles, Donghua University, Shanghai, 201620, China; $b$ : Key Laboratory of Textile Science \& Technology, Ministry of Education, Donghua University, Shanghai, 201620, China; c: Innovation Center for Textile Science and Technology, Donghua University, Shanghai, 201620, P.R. China; *Corresponding author: yucw@dhu.edu.cn

\section{INTRODUCTION}

Linen is a type of natural textile fiber with good moisture absorption, heat dissipation, and antibacterial and anti-static properties (He et al. 2005). Linen has global applications (Yu et al. 1997) because it is stiff and comfortable to wear, etc. Viscose, a kind of cellulose fiber, is made from natural cellulose (pulp) and spun by regeneration from cellulose xanthate solution (Dong et al. 2009). Viscose fiber has the advantages of easy dyeing, softness, good draping, and comfortable wear.

Common analytical methods for detecting fiber content in fabrics are based on microscopy or specific component dissolution by formic acid/ zinc chloride chemical method (GB/T 2910.6-2009). However, these analytical methods are time-consuming, costly, and not environmentally friendly. Therefore, the development of a rapid, accurate and environmentally friendly approach is necessary. In recent years, near-infrared (NIR) spectroscopy has been an effective method for quantitative and qualitative composition analysis. In this study, NIR was employed to analyze the linen content in linen/viscose blends quantitatively.

Near-infrared light refers to the electromagnetic wave whose wavelength is between the visible light region and the mid-infrared region. The near-infrared spectrum region defined by the American Society for Testing and Materials (ASTM) (ASTM E 1944-

Huang and Yu (2020). "Fiber content analysis via NIR," BioResources 15(2), 3006-3016. 3006 
1998) is the region of 780 to $2526 \mathrm{~nm}$, and the wave number range is 12820.5 to 3958.8 $\mathrm{cm}^{-1}$. The absorption band in the near-infrared light region is mainly generated by lowenergy electron transitions and the multiple frequency and combined frequency absorption of the stretching vibration of hydrogen groups (such as $\mathrm{N}-\mathrm{H}, \mathrm{O}-\mathrm{H}$, and $\mathrm{C}-\mathrm{H}$ ). The most important use of this optical region is to conduct quantitative analysis of some substances, which is widely used in the detection of organic compounds in agricultural products, petroleum, and other fields. The NIR technology can be used for non-destructive, rapid, and multi-component simultaneous analysis under complex background without side effects such as chemical reagent consumption and pollution (Zhang et al. 2012).

NIR techniques have been applied in cotton, silk, wool, and cashmere identification (Chen et al. 2006, 2007; Wu et al. 2008; Yan et al. 2013). The significant difference in NIR spectra of various fibers allows the accurate analysis of cotton/polyester and cotton/ wool blends (Feng et al. 2005; Liu et al. 2013). The fiber content in wool/cashmere blends has been measured with standard prediction errors less than 3\% (Zhou et al. 2017). A previous study on the fiber content identification in linen/cotton blends failed to determine the characteristic wavelength bands or duplicate the data both validation and calibration sets (Miryeong et al. 2005).

Thus, due to similar molecular structures and geometrical attributes (Zhou et al. 2017), measuring one fiber contents in a cellulose or protein blended sample such as linen/viscose is worth further study. This study was aimed at developing a reliable, convenient, and fast method to determine linen content in linen/viscose blends based on NIR spectroscopy.

\section{EXPERIMENTAL}

\section{Sample Preparation}

The linen fibers and viscose fibers were provided by Royal Golden Eagle Co., Ltd. (Zhejiang Province, China). The fibers were ground to powder (micron level) using a planetary ball mill (Miryeong et al. 2005; Baheti et al. 2012; Zhao 2017) (QM-3SP2L) with zirconia balls ( $\$ 5 \mathrm{~mm}$ ) for $4 \mathrm{~h}$. A series of linen/viscose fibers with increasing ratio of linen $(0 \%, 10 \%, 20 \%, 30 \%, 40 \%, 50 \%, 60 \%, 70 \%, 80 \%, 90 \%$, and $100 \%)$ were fabricated.

\section{Spectra Collection and Pre-treatment}

The spectrometer (Thermo Antaris, Waltham, MA, USA) used in this study has a spectrum range of 12500 to $4000 \mathrm{~cm}^{-1}$ with a spectral resolution of $8 \mathrm{~cm}^{-1}$. Each spectrum obtained in diffuse reflection was the average of 32 scans. After achieving hygroscopic equilibrium, about $1 \mathrm{~g}$ sample was used for scanning. Remixing, repacking, and rescanning were applied to reduce the error caused by uneven mixing, and each sample was scanned 60 times.

In terms of spectral analysis, the data included the composition and structural information of tested samples and the noise including measurement error, interference between different components, etc. Proper spectral pretreatment or transformation on the original near-infrared spectrum could weaken the influence of various non-target factors, remove irrelevant information variables, and enhance the resolution and sensitivity, resulting in improved prediction ability and calibration model stability (Chu et al. 2004).

Huang and Yu (2020). "Fiber content analysis via NIR," BioResources 15(2), 3006-3016. 3007 
Through changing testing locations, it was found that the spectral fluctuations of the pure linen samples was obvious, as shown in Fig. 1(a). Similar spectral fluctuations occurred in all other samples.
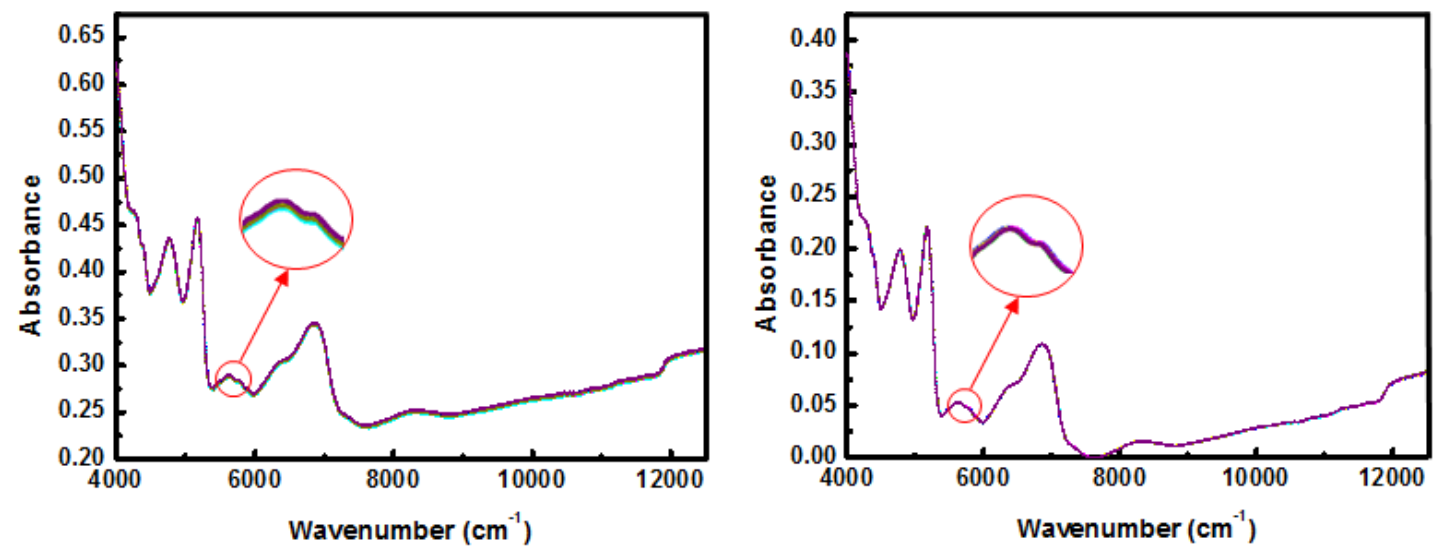

Fig. 1. Spectra of (a) original (b) pretreated pure linen sample (10 times scanned)

To ensure the spectral accuracy, appropriate spectral pretreatment methods, such as smoothing, baseline offset, multiplicative scatter correction, etc., should be employed to reduce the interference of specific physical factors (Balabin and Smirnov 2011; Manley 2014). By using smoothing processing, the high frequency noise can be effectively reduced, the signal-to-noise ratio can be improved, and the smooth spectral image can be obtained. Baseline offset is a centralized process that eliminates the influence of instrument status and test environment on the diffuse reflection process. Multiplicative scatter correction offers a simple technique to minimize the effect of scattering caused by the uneven distribution of sample particle size and spacing.

In this work, the tested near-infrared spectra were processed by smoothing, baseline offset, and multiplicative scattering correction. As shown in Fig. 1(b), the pretreated spectra of the linen fibers overlapped together, eliminating the errors caused by test conditions, sample particle size and morphology, etc.

\section{Characteristic Wavelength Bands Selection}

Figure 2 displays the mean spectral of linen and viscose, respectively, showing similar spectra characteristics. There were identical spectra peaks and wave fluctuation within 8200 to $4000 \mathrm{~cm}^{-1}$ bands, suggesting their analogous chemical structures. The difference of absorption intensity of absorption band is related to the content of molecular composition or chemical group, which can be used to further determine the ratios of linen/viscose blends.

In the spectral range of 12500 to $8200 \mathrm{~cm}^{-1}$ the two fibers showed a different variation trend of absorbance, attributed to their color difference, which is consistent with previous findings (Miryeong et al. 2005).

To identify the characteristic chemical bond positions and the wavelength range, the peak position and peak range should be determined. In the spectra processed by the second derivative, the maximum value in negative direction is stand for the peak value of the original spectra. The peak range is determined by spectra inflection point, which is the null point of the second derivative. 


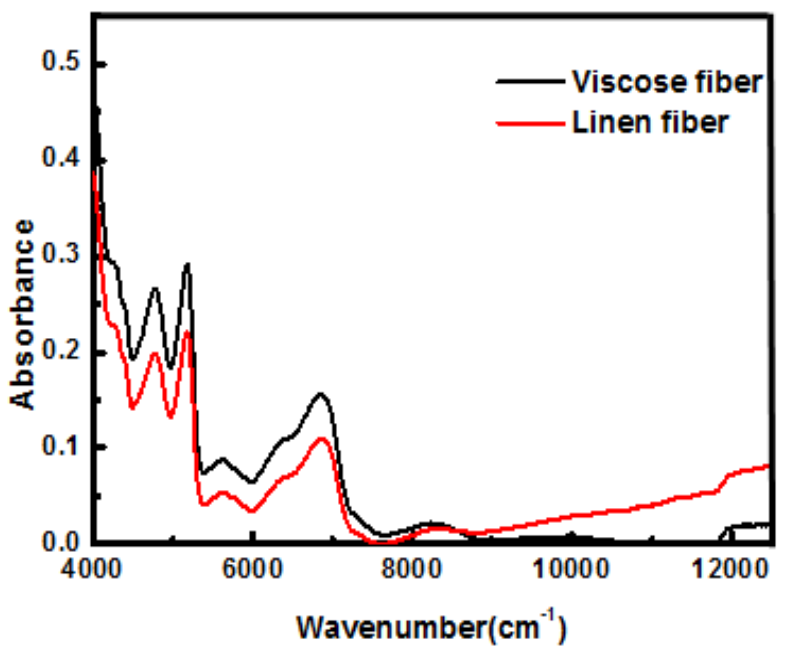

Fig. 2. Spectra of linen and viscose samples

As shown in Fig. 3(a), the second derivative spectra of linen and viscose were consistent with the results shown in Fig. 2. Differences in their spectra were specific to wavenumbers in the band range of 8000 to $4000 \mathrm{~cm}^{-1}$. In the 12500 to $8000 \mathrm{~cm}^{-1}$ range, there was little fluctuation. In the wavenumber range 8000 to $4000 \mathrm{~cm}^{-1}$, there were differences between the spectra of linen and viscose in some specific wavenumbers, while in 12500 to $8000 \mathrm{~cm}^{-1}$ range, there was little fluctuation. In Fig. 3(b), the absorbance of viscose fiber is higher than that of linen fiber in wavenumbers of $4038 \mathrm{~cm}^{-1}, 4304 \mathrm{~cm}^{-1}$, $4404 \mathrm{~cm}^{-1}, 4786 \mathrm{~cm}^{-1}, 5199 \mathrm{~cm}^{-1}, 5634 \mathrm{~cm}^{-1}, 5916 \mathrm{~cm}^{-1}$, and $6977 \mathrm{~cm}^{-1}$, attributed to higher cellulose content in viscose than in linen. The absorbance of linen in wavenumbers of 4264 and $5795 \mathrm{~cm}^{-1}$ was higher than that of viscose, and the possible reason was that these two wavenumbers correspond to the stretching deformation mode of $\mathrm{C}-\mathrm{H}$ bond (Schwanninger et al. 2011) of wax and lignin in linen fiber. In this study, 14 zero points were identified around ten crests, which was the inflection point in Fig. 2, and then seven characteristic spectral bands were fixed. Table 1 details the vibrational forms of molecular bonds (Schwanninger et al. 2011) corresponding to the seven characteristic spectral bands.

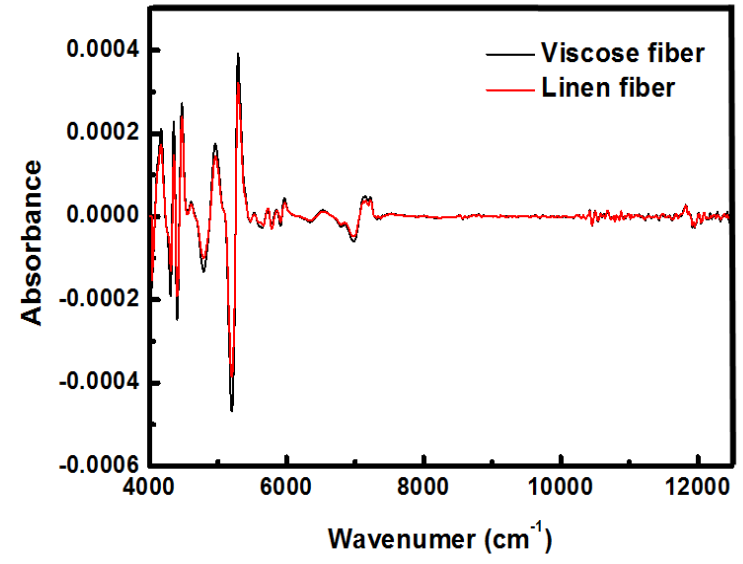

(a) 12500 to $4000 \mathrm{~cm}^{-1}$

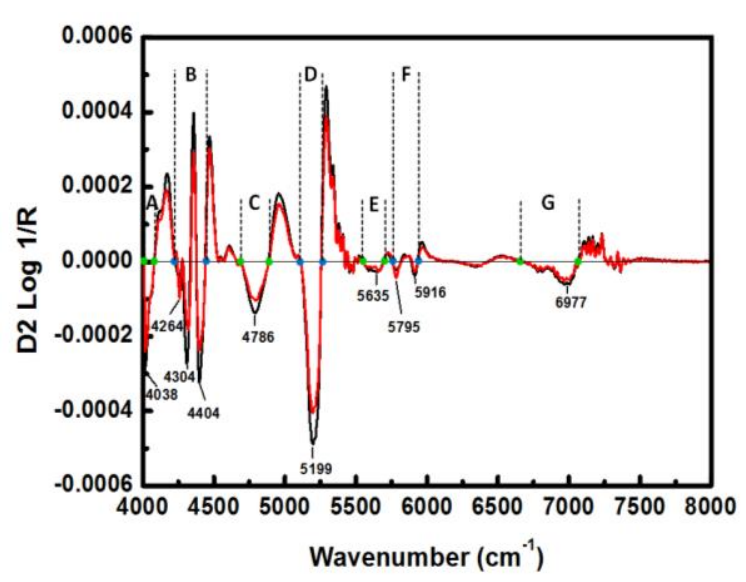

(b) 8000 to $4000 \mathrm{~cm}^{-1}$

Fig. 3. The second derivative spectra of linen and viscose in spectral range (a) 12500 to 4000 $\mathrm{cm}^{-1}$ and (b) 8000 to $4000 \mathrm{~cm}^{-1}$ 
Table 1. Seven Characteristic Spectral Waveband and Vibrational Forms of Molecular Bond

\begin{tabular}{|c|c|c|}
\hline No. & Spectral Band $\left(\mathrm{cm}^{-1}\right)$ & Vibrational Forms of Molecular Bond \\
\hline A & $4080-4000$ & C-H str. + C-C str. \\
\hline B & $4442-4227$ & $\begin{array}{l}\text { C-H str.+ C-H def., C-H str.+ C- } \mathrm{H}_{2} \text { def., O-H str. + C-O str., C- } \\
\mathrm{H}_{2} \text { str.+ } \mathrm{C}-\mathrm{H}_{2} \text { def., O-H str. + C-C str., } 2^{\text {nd }} \mathrm{OT} \mathrm{C}-\mathrm{H}_{2} \text { bend. }\end{array}$ \\
\hline C & $4891-4658$ & $\begin{array}{l}\mathrm{C}-\mathrm{H} \text { str.+ } \mathrm{C}=\mathrm{O} \text { str., C-H str.+ } \mathrm{C}=\mathrm{C} \text { str., O-H def. + O-H str., C-H } \\
\text { def. + O-H str. }\end{array}$ \\
\hline $\mathrm{D}$ & $5253-5110$ & $\mathrm{O}-\mathrm{H}$ asym. str. $+\mathrm{O}-\mathrm{H}$ def. of $\mathrm{H}_{2} \mathrm{O}$ \\
\hline $\mathrm{E}$ & $5701-5556$ & $1^{\text {st }}$ OT C-H str., $1^{\text {st }}$ OT C-H 2 str. \\
\hline $\mathrm{F}$ & $5936-5756$ & $1^{\text {st }} \mathrm{OT} \mathrm{C}-\mathrm{H}$ str. \\
\hline G & 7067-6666 & $\begin{array}{l}1^{\text {st }} \text { OT O-H str., } 1^{\text {st }} \text { OT C-H str.+ C-H def., } 1^{\text {st }} \text { OT C-H str.+ C-H } \\
\text { bend. }\end{array}$ \\
\hline
\end{tabular}

*Str.: stretching vibration; def.: deformation vibration; +: combination band; bend: bending vibration; $1^{\text {st }} \mathrm{OT}$ : first overtone; $2^{\text {nd }} \mathrm{OT}$ : second overtone

As shown in Table 1, the molecular vibration forms of these seven characteristic bands are closely related to the chemical bonds of cellulose. Therefore, these bands were all selected as characteristic spectral segments for spectral data analysis in this study.

\section{Near-Infrared Data Analysis}

The Unscrambler 10.5 X software (CAMO, Oslo, Norway) was used to develop the chemometric models. Partial least squares (PLS) regression is a multivariate statistical analysis method used to build NIR models (Zhang et al. 2012). The PLS method decomposes the variables $\mathrm{X}$ and $\mathrm{Y}$ as follows,

$$
\begin{aligned}
& X=T P+E \\
& Y=U Q+F
\end{aligned}
$$

where $X$ is an $\mathrm{n}^{*} \mathrm{~m}$ matrix of predictors, $Y$ is an $\mathrm{n} * \mathrm{p}$ matrix of responses; $T$ and $U$ are $\mathrm{n} * 1$ matrices that are, respectively, projections of $X$ (the $X$ score, component or factor matrix) and projections of $Y$ (the $Y$ scores); $P$ and $Q$ are, respectively, $\mathrm{m}^{* 1}$ and $\mathrm{p}^{* 1}$ orthogonal loading matrices; and matrices $E$ and $F$ are the error terms, assumed to be independent and identically distributed random normal variables. The decompositions of $X$ and $Y$ are made so as to maximise the covariance between $T$ and $U$. The second part is the linear regression of $T$ and $U$, defined as follows.

$$
\begin{aligned}
& U=T B \\
& B=\left(T^{T} T\right)^{-1} T^{T} Y
\end{aligned}
$$

In the prediction, the score $T$ of the spectral matrix $X$ above was first calculated according to $P$, and then the prediction was realized by $Y=T B Q$. In the real PLS algorithm, the matrix decomposition and regression are combined into one part, that is, the $X$ and $Y$ matrix decomposition is carried out at the same time, and the information of $Y$ is introduced into the $X$ matrix decomposition process, and $X$ is converted into $X$ before each new principal component is calculated. In this way, the principal component of $X$ is directly associated with $Y$.

In this paper, PLS regression method was used to find the optimum relationship between the spectral data matrix and the properties of interest (Feng et al. 2005). The PLS model of the relationship between spectral characteristics and component content was established by using chemometric analysis. The accuracy and the prediction ability of the 
PLS model were tested by internal cross-validation of the calibration sample set and external validation of the validation sample set. Six of eleven samples with fiber contents of $0 \%, 20 \%, 40 \%, 60 \%, 80 \%$, and $100 \%$ were chosen as the calibration subset, and five samples with fiber contents of $10 \%, 30 \%, 50 \%, 70 \%$, and $90 \%$ as the validation subset.

\section{RESULTS AND DISCUSSION}

Figure 4 displays spectra of the calibration subsets with different LCs (linen contents). The absorbance change in the seven characteristic spectral bands (A: 4000 to $4080 \mathrm{~cm}^{-1}$, B: 4227 to $4442 \mathrm{~cm}^{-1}$, C: 4658 to $4891 \mathrm{~cm}^{-1}$, D: 5110 to $5253 \mathrm{~cm}^{-1}$, E: 5556 to $5701 \mathrm{~cm}^{-1}$, F: 5756 to $5936 \mathrm{~cm}^{-1}$, G: 6666 to $7067 \mathrm{~cm}^{-1}$ ) were marked by red rectangles. With the increase of the linen content of linen/viscose blended fiber, the absorbance of the seven characteristic bands decreased gradually. The sequentiality and recursion of spectral arrangement will be beneficial to the fiber content determination.

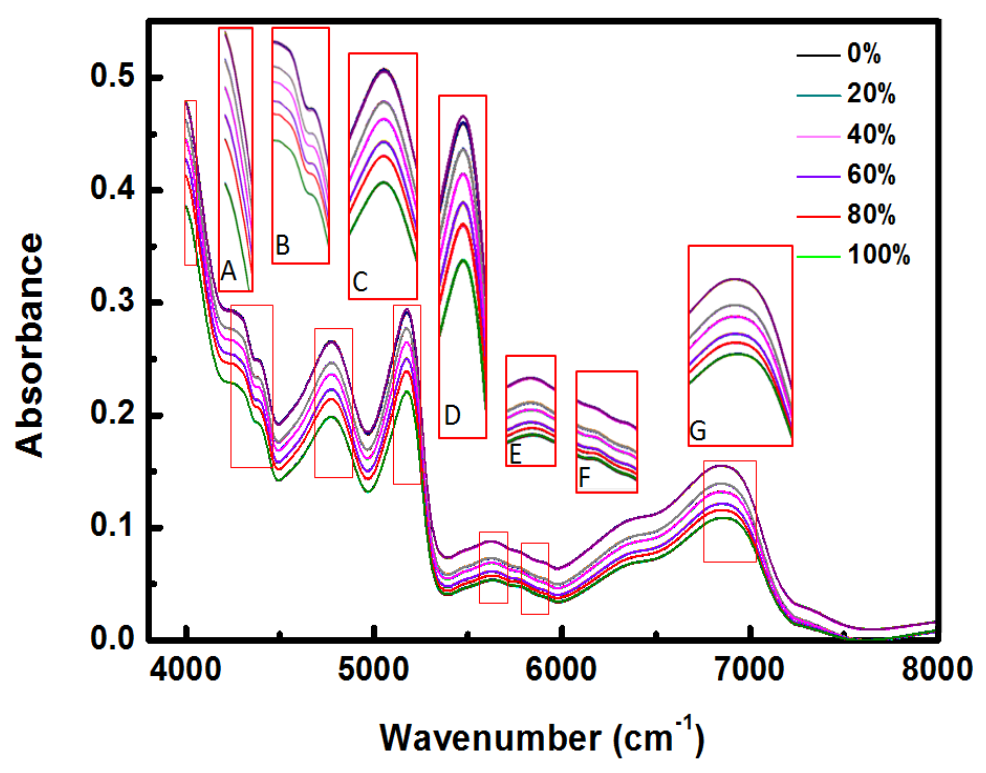

Fig. 4 Spectra of the calibration subsets with different LCs

To reduce the heterogeneity of blended fibers, 60 scans were taken for each sample. An average of 1 curve was taken for every 6 values; a curve corresponds to a set of spectra. Thus, for each sample there were 10 sets of spectra. In total, 110 spectra sets were established, in which 60 spectra sets for calibration and 50 spectra sets for validation of NIR models.

The effectiveness of a model was evaluated (Miryeong et al. 2005; Huang et al. 2019) by coefficient of multiple determination for calibration $\left(\mathrm{Rc}^{2}\right)$, coefficient of multiple determination for prediction $\left(\mathrm{Rp}^{2}\right)$, root mean square error of calibration (RMSEC), root mean square error of prediction (RMSEP), and ratio of performance to standard deviate (RPD).

$$
\operatorname{Rc}^{2}=1-\frac{\sum_{i=1}^{n_{c}}\left(y_{i}-\hat{y}_{i}\right)^{2}}{\sum_{i=1}^{n_{C}}\left(y_{i}-\bar{y}_{i}\right)^{2}}
$$




$$
\begin{aligned}
& \operatorname{Rp}^{2}=1-\frac{\sum_{i=1}^{n_{p}}\left(y_{i}-\hat{y}_{i}\right)^{2}}{\sum_{i=1}^{n_{p}}\left(y_{i}-\bar{y}_{i}\right)^{2}} \\
& \mathrm{RMSEC}=\sqrt{\frac{\sum_{i=1}^{n_{c}}\left(\hat{y}_{i}-y_{i}\right)^{2}}{n_{c}}} \\
& \mathrm{RMSEP}=\sqrt{\frac{\sum_{i=1}^{n_{p}}\left(\hat{y}_{i}-y_{i}\right)^{2}}{n_{p}}}
\end{aligned}
$$

where $n_{c}$ and $n_{p}$ are the number of samples in the calibration set and validation set, respectively, $\mathrm{y}_{\mathrm{i}}$ is the measured value for the $\mathrm{i}^{\text {th }}$ sample, $\hat{y}_{i}$ is the predicted value for the $\mathrm{i}^{\text {th }}$ sample, $\bar{y}_{i}$ is the mean of $\mathrm{y}_{\mathrm{i}}$ for all the samples. A higher R-value (close to 1 ) indicates a good fit. A good model not only has a small value of RMSEC and RMSEP, but also a smaller difference between them.

The RPD was calculated to assess the predictive ability of the NIR model (Huang et al. 2019). Generally, an RPD value greater than 2 indicates that the model has prediction ability in some extent. An RPD value greater than 3 indicates that the model has excellent prediction ability (Williams and Sobering 1993; Fagan et al. 2011). The higher value of RPD indicates a more accurate model predictive ability. The RPD was calculated as follows,

$$
\mathrm{RPD}=\frac{\sqrt{\sum_{i=1}^{n_{p}}\left(y_{i, p}-\bar{y}_{i, p}\right)^{2} /\left(n_{p}-1\right)}}{R M S E P}
$$

where $\mathrm{n}_{\mathrm{p}}$ is the number of samples in validation set, $y_{i, p}$ is the true value for the $\mathrm{i}^{\text {th }}$ sample in validation set, $\bar{y}_{i, p}$ is the mean of $y_{i, p}$ for all the samples in validation set.

The data in Table 2 suggest that the reduced range had significantly better model fitness than the full range model, with $\mathrm{Rc}^{2}$ of $0.995, \mathrm{Rp}^{2}$ of 0.998 , RMSEC of $2.432 \%$, RMSEP of $1.182 \%$, and RPD of 24.336 .

Table 2. Full Range Versus Reduced Range Model Results

\begin{tabular}{|c|c|c|}
\hline & Full Range & Reduced Range \\
\hline Rc $^{2}$ & 0.9998 & 0.995 \\
\hline RMSEC & 0.444 & 2.432 \\
\hline RMSECV & 0.462 & 2.530 \\
\hline RP $^{2}$ & 0.979 & 0.998 \\
\hline RMSEP & 4.031 & 1.182 \\
\hline RPD & 7.504 & 24.336 \\
\hline
\end{tabular}

Figure 5 displays plots of residual versus referenced LCs and predicted versus referenced LCs of the calibration subsets. In Fig. 5(a), the predicted LCs were in good agreement with the reference values with residuals under 6\%. In Fig. 5(b), the predicted versus referenced plots shows the slope of linear equation was close to 1 , in which $y$ is the reference LC and $\hat{y}$ is the predicted LC. The predicted value was 0.996, and the offset was 0.204 , indicating a high quality of the PLS model. 

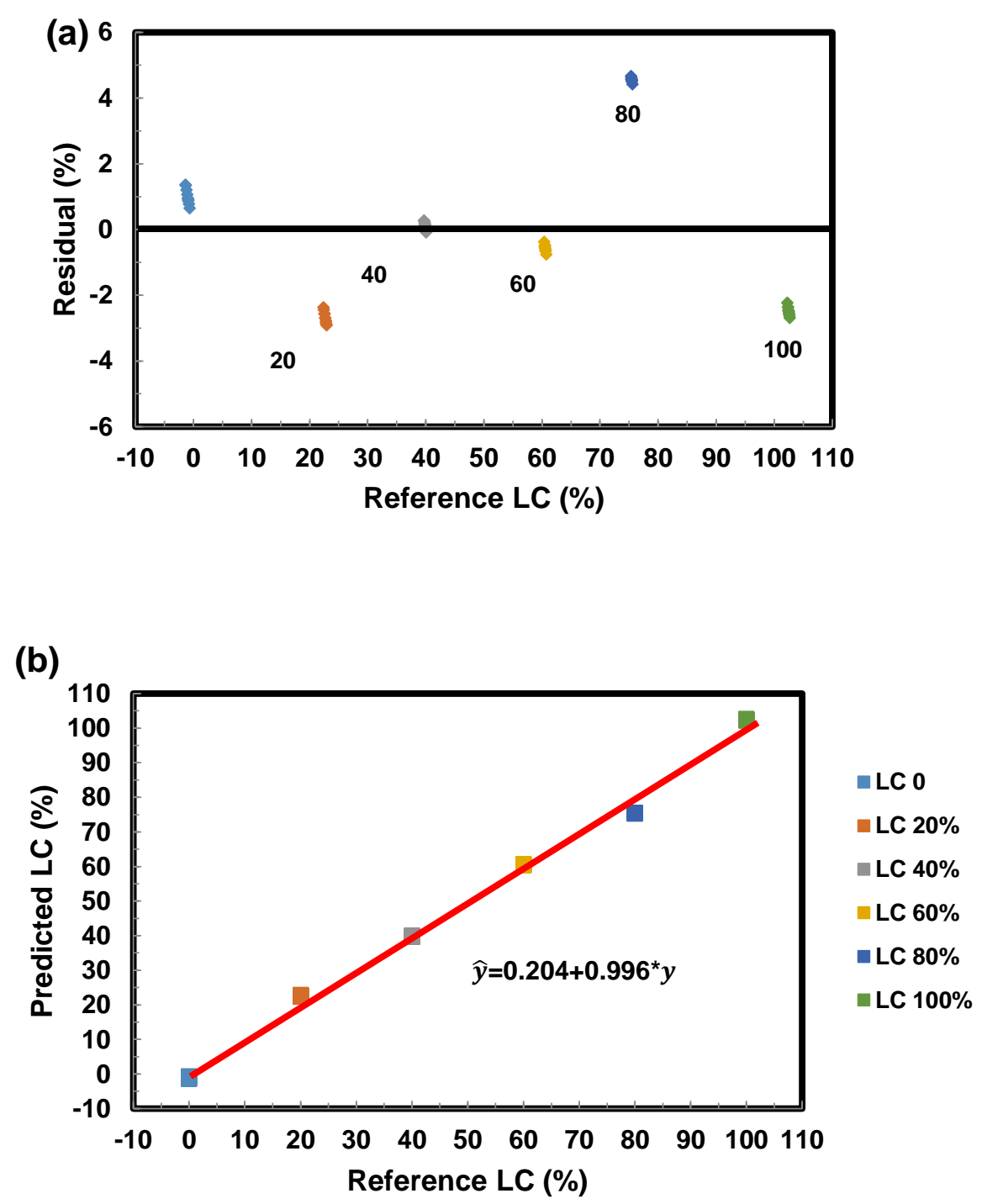

Fig. 5. (a) Plots of residual versus referenced LCs of the calibration subsets; (b) PLS regression plots of predicted versus referenced LCs of the calibration subsets 


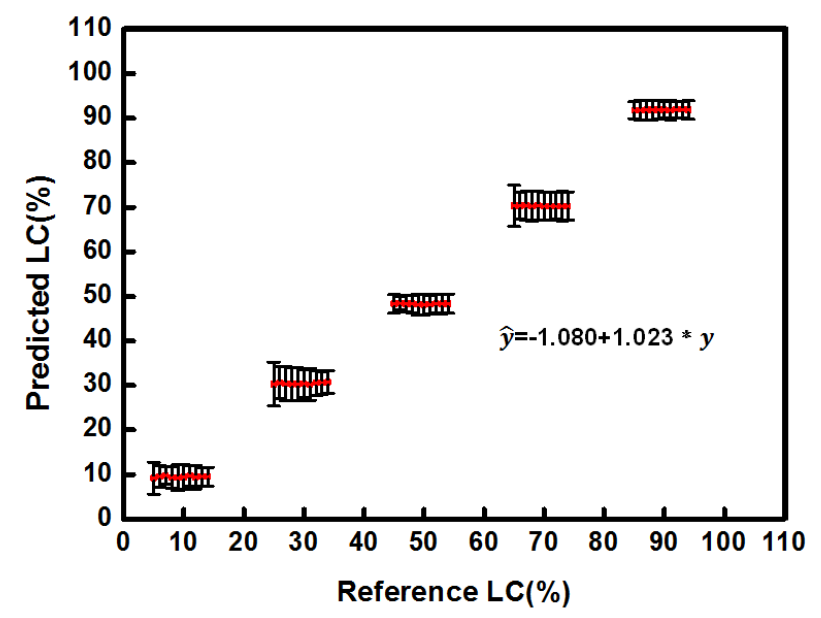

Fig. 6 Predicted versus referenced LCs in validation subsets

Figure 6 shows the predicted versus referenced LCs in the validation subsets, and Table 3 shows the ten predicted values of every validation sample, in which the SD value was very low. The samples for validation represented different LCs from those for calibration, but with consistent results. The PLS model in the validation subsets provided accurate prediction with $\mathrm{Rp}^{2}$ of 0.998 , RMSEC of $2.432 \%$, RMSEP of $1.182 \%$, and RPD of 24.336 .

Table 3. Prediction Results of LCs in Validation Subsets

\begin{tabular}{|c|c|c|c|c|c|}
\hline \multirow{2}{*}{ Predicted LC (\%) } & \multicolumn{5}{|c|}{ LC (\%) } \\
\cline { 2 - 5 } & 10 & 30 & 50 & 70 & 90 \\
\hline 1 & 9.20 & 30.31 & 48.32 & 70.31 & 91.79 \\
\hline 2 & 9.58 & 30.57 & 48.47 & 70.33 & 91.79 \\
\hline 3 & 9.82 & 30.35 & 48.30 & 70.35 & 91.83 \\
\hline 4 & 9.33 & 30.29 & 48.34 & 70.27 & 91.86 \\
\hline 5 & 9.28 & 30.30 & 48.16 & 70.39 & 91.89 \\
\hline 6 & 9.38 & 30.46 & 48.15 & 70.28 & 91.88 \\
\hline 7 & 9.69 & 30.22 & 48.16 & 70.25 & 91.80 \\
\hline 8 & 9.39 & 30.55 & 48.37 & 70.26 & 91.94 \\
\hline 9 & 9.56 & 30.60 & 48.31 & 70.28 & 91.93 \\
\hline 10 & 9.54 & 30.76 & 48.34 & 70.27 & 91.85 \\
\hline Mean & 9.48 & 30.44 & 48.29 & 70.30 & 91.86 \\
\hline SD & 0.19 & 0.18 & 0.10 & 0.04 & 0.05 \\
\hline
\end{tabular}

\section{CONCLUSIONS}

1. A near infrared (NIR) spectroscopic method was applied to determine the fiber contents of linen/viscose blends conveniently and accurately. A series of linen/viscose samples with varied weight ratios were prepared. Through remixing, repacking, and rescanning, the NIR spectra of all the samples were collected.

2. The spectra was pretreated by smoothing, baseline offset and multiplicative scattering correction to eliminate the errors caused by test conditions, sample particle size and morphology. 
3. Seven characteristic spectral bands which were closely related to the chemical bonds of cellulose were identified: A: 4000 to $4080 \mathrm{~cm}^{-1}$, B: 4227 to $4442 \mathrm{~cm}^{-1}$, C: 4658 to $4891 \mathrm{~cm}^{-1}$, D: 5110 to $5253 \mathrm{~cm}^{-1}$, E: 5556 to $5701 \mathrm{~cm}^{-1}, \mathrm{~F}: 5756$ to $5936 \mathrm{~cm}^{-1}$, G: 6666 to $7067 \mathrm{~cm}^{-1}$.

4. Comparing with the evaluation indexes of the effect of full range and characteristic spectral range models, the reduced range demonstrated better model fitness than full range model, as demonstrated by similar $\mathrm{Rc}^{2}(0.995)$, larger $\mathrm{Rp}^{2}(0.998)$ and $\mathrm{RPD}$ (24.336), also smaller difference between RMSEC (2.432\%) and RMSEP (1.182\%).

5. The predicted versus referenced plots of LCs shows the slope of linear equation was 0.996 , and the offset was 0.204 . The ten predicted values of each validation sample shows low SD value $(<0.2)$.

6. The high quality PLS model has been proved to be an accurate and effective method for determining the linen contents in linen/viscose blends.

\section{ACKNOWLEDGMENTS}

The authors are grateful for the support of the China Agriculture Research System for Bast and Leaf Fiber Crops: CARS-16.

\section{REFERENCES CITED}

ASTM E 1944-1998 (2007). "Standard practice for describing and measuring performance of laboratory Fourier transform near-infrared (FT-NIR) spectrometers: Level zero and level one tests," ASTM International, West Conshohocken, PA, USA.

Balabin, R. M., and Smirnov, S. V. (2011). "Variable selection in near-infrared spectroscopy: Benchmarking of feature selection methods on biodiesel data," Analytica Chimica Acta 692(1-2), 63-72. DOI: 10.1016/j.aca.2011.03.006

Baheti, V. K., Abbasi, R., and Militky, J. (2012). "Ball milling of jute fibre wastes to prepare nanocellulose," World Journal of Engineering 9(1), 45-50. DOI: 10.1260/1708-5284.9.1.45

Chen, B., Cui, G., Jin, S. Z., and Wang, X. T. (2007). "Application of near infrared spectra in rapid inspection of cotton contents," Journal of Jiangsu University 28(3), 185-188.

Chen, B., Wu, X. T., and Ni, K. (2006). "Application of correlation analysis in rapid detecting silk content of fabric textile by NIR," Spectroscopy Instrument and Analysis 1, 52.

Chu, X. W., Yuan, H. F., and Lu, W. Z. (2004). "Progress and application of spectral pretreatment and wavelength selection methods in NIR analysis," Chemical Progress 7(16), 528-543.

Dong, H. W. (2009). "Modern chemical fiber- viscose fiber," Guangxi Journal of Light Industry (1), 96-101.

Fagan, C. C., Everard, C. D., and McDonnell, K. (2011). "Prediction of moisture, calorific value, ash and carbon content of two dedicated bioenergy crops using near-

Huang and Yu (2020). "Fiber content analysis via NIR," BioResources 15(2), 3006-3016. 3015 
infrared spectroscopy," Bioresource Technology 102(8), 5200-5206. DOI:

10.1016/j.biortech.2011.01.087

Feng, H. N., Gan, B., and Jin, S. Z. (2005). "Concentration test of textile mixture of cotton and terlene with NIR method," Laser \& Infrared 35, 768.

GB/T 2910.6-2009. "Textile- Quantitative chemical analysis- Part 6: Mixtures of viscose or certain types of cupro or modal or lyocell and cotton fibres (method using formic acid and zinc chloride," The State Bureau of Quality and Technical Supervision, Beijing, China.

He, J., Wu, L. L., and Yu, J. Y. (2005). "Study on fine modification of flax fiber," Journal of Textile Research 26(2), 99-102.

Huang, J., and Yu, C. W. (2019). "Determination of cellulose, hemicellulose and lignin content using near-infrared spectroscopy in flax fiber," Textile Research Journal 89(23-24), 4875-4883. DOI: 10.1177/0040517519843464

Liu, L., Yan, L., and Xie, Y. (2013). "Determination of fiber contents in blended textiles by NIR combined with BP neural network," Int. Scholarly Res. Not. Textile 546481, 1-5. DOI: $10.1155 / 2013 / 546481$

Manley, M. (2014). "Near-infrared spectroscopy and hyperspectral imaging: Nondestructive analysis of biological materials," Chemical Society Reviews 43(24), 8200. DOI: 10.1039/C4CS00062E

Miryeong, S., David, S. H., Danny, E. A., and Franklin, E. B. (2005). "Fourier transform near-infrared spectroscopy for determining linen content in linen/cotton blend products," Textile Research Journal 75(8), 583-590. DOI: 10.1177/0040517505057167

Schwanninger, M., Rodrigues, J.C., and Fackler, K. (2011). "A review of band assignments in near infrared spectra of wood and wood components," Journal of Near Infrared Spectroscopy 19(5), 287-308. DOI: 10.1255/jnirs.955

Williams, P., and Sobering, D. (1993). "Comparison of commercial near infrared transmittance and reflectance instruments for analysis of whole grains and seeds," Journal of Near Infrared Spectroscopy 1(1), 25. DOI: 10.1255/jnirs.3

Wu, G. F., Zhu, D. S., and He, Y. (2008). "Identification of fine wool and cashmere by using Vis/NIR spectroscopy technology," Spectroscopy and Spectral Analysis 28(6), 1260-1263. DOI: 10.1117/12.791231

Yan, J. J., Nicole, V., and Xu, B. G. (2013). "Characterization of degradation of cotton cellulosic fibers through near infrared spectroscopy," Journal of Polymers and the Environment 21(4), 902-909. DOI: 10.1007/s10924-013-0605-z

Yu, C. Y., and Xia, J. Y. (1997). “Technology of flax spinning," Heilongjiang Science \& Technology Press, 23-25.

Zhang, X. C., Wu, J. S., and Xu, Y. (2012). "Near infrared spectroscopy and its application in modern agriculture," Publishing House of Electronics Industry, 19-20.

Zhao, K. F. (2017). "Study on determination of adulteration in matcha based on the nearinfrared spectroscopy," Masteral dissertation of Anhui Agriculture University, 12.

Zhou, J. F., Wang, R. W., Wu, X. Y., and Xu, B. G. (2017). "Fiber-content measurement of wool-cashmere blends using near-infrared spectroscopy," Applied Spectroscopy 71(10), 2367-2376. DOI: 10.1177/0003702817713480

Article submitted: October 22, 2019; Peer review completed: January 23, 2020; Revised version received and accepted: March 5, 2020; Published: March 13, 2020.

DOI: $10.15376 /$ biores. 15.2.3006-3016

Huang and Yu (2020). "Fiber content analysis via NIR," BioResources 15(2), 3006-3016. 3016 